\title{
The Effects of Cervical Stabilization Exercises with Breathing Exercises on Respiratory Function in Subjects with Forward Head Posture
}

\author{
LIJUN YU \\ Department of Rehabilitation Science, Graduate School of Daegu University, \\ Gyeongsangbuk-do Province, SOUTH KOREA \\ QIAN GU \\ Department of Rehabilitation Science, Graduate School of Daegu University, \\ Gyeongsangbuk-do Province, SOUTH KOREA \\ TAEHO KIM* \\ Department of Physical Therapy, Daegu University, \\ Gyeongsangbuk-do Province, SOUTH KOREA \\ *Corresponding author : TaeHo Kim
}

\begin{abstract}
This study aimed to compare the effects of cervical stabilization exercises with breathing exercises and cervical stabilization exercises with thoracic spine extension exercises on respiratory function in subjects with forward head posture (FHP). The study included 30 adults with FHP. They were divided into two groupsexperimental (EG) and control (CG) - with 15 participants in each group. The EG was asked to perform cervical stabilization exercises with breathing exercises, and the $\mathrm{CG}$ was asked to perform cervical stabilization exercises with thoracic spine extension exercises. After 4 weeks of exercise, the changes in respiratory function were measured again. The results showed significant differences in the EG before and after exercise in terms of forced vital capacity (FVC), forced expiratory volume in 1 second (FEV1), peak expiratory flow (PEF), and forced vital capacity/vital capacity max $(\mathrm{FVC} / \mathrm{VCmax} \%)(\mathrm{p}<0.05)$. The CG showed only a significant increase in FVC, $\mathrm{FEV} 1$, and $\mathrm{FVC} / \mathrm{VCmax} \%$ after the experiment $(\mathrm{p}<0.05)$. In the between-group comparison, there were significant differences noted in FVC, FEV1, PEF, and FVC/VCmax\% in the EG compared to the CG $(\mathrm{p}<0.05)$. As a result, cervical stabilization exercises with breathing exercises are recommended to promote respiratory function.
\end{abstract}

Key-Words: - Breathing exercises, Cervical stabilization, Forward head posture, Thoracic extension.

Received: January 17, 2021. Revised: August 18, 2021. Accepted: August 29, 2021. Published: September 13, 2021.

\section{Introduction}

With the increased use of handheld electronic devices, there has been a rise in the number of people who are prone to poor sitting postures, such as forward head posture (FHP) [1]. FHP can be defined as an abnormal state in which the head lies in front of the gravity line around the sagittal view, with added extension of the upper cervical spine and increased flexion of the lower cervical and upper thoracic spine [2].

Different bones can form different morphological structures to provide support, help exercise, and protect important internal organs [3]. Such as cervical vertebrae, thoracic vertebrae. FHP has negative effects on alveolar ventilation and expansion of the thorax, thereby reducing vital capacity and lung volume and weakening respiratory function. It decreases muscle power in the neck, which is followed by a reduction in the muscle power of the respiratory muscles. Therefore, injury to the respiratory muscles is associated with damage to motor control in the cervical spine. The loss of respiratory function has a connection with functional disorders associated with the weakening of the respiratory muscles and trunk posture [4]. FHP is caused by decreased physical activity and muscle fatigue. The weakness of the respiratory muscles 
decreases lung capacity [5]. The shortness of accessory respiratory muscles affects respiratory function [6]. A study reported that FHP changes the alignment of the rib cage and thoracic spine because of a bent posture, thus causing respiratory dysfunction [6]. FHP has been reported to elevate the rib cage and reduce diaphragm contraction and thoracoabdominal mobility [7]. It can cause excessive kyphosis of the thoracic spine and increase kyphosis because FHP can reduce thoracic mobility, which has a negative effect on respiratory capabilities [4].

Previous research showed that the smaller the craniovertebral angle (CVA) in FHP, the worse the respiratory and circulatory functions [2]. Several studies have assessed the carriage of the body in mouth-breathing subjects, and the common view is that FHP is the principal variation. FHP brings about disorganization of the muscle blocks (posterior, anterior, and transverse muscles) and damages diaphragmatic mobility and function [8]. The weakness of the deep neck extensor and flexor muscles results in reduced stability of the thoracic and cervical spine along with changes in the mechanics of the rib cage [9]. Over a long period, FHP shortens and weakens these muscles, leading to decreased breathing [10]. The shortening and weakness of the neck muscles have an effect not only on the pain and limitation of movement, but also on the lung volume and diameter of the thoracic cage, which can cause dysfunction of the respiratory muscles, such as the intercostal and diaphragm muscles [2]. Pain affects their daily work to a medium or large extent [11].

Breathing exercises include pursed-lip breathing, diaphragmatic breathing (also known as abdominal breathing or breathing control), active expiration, pranayama yoga (comprising timed breathing techniques, with a focus on ventilation feedback training), and expiration, where participants aim to achieve individualized goals for respiratory rate and pattern with computerized feedback [12]. These exercises aim to alter respiratory muscle recruitment, lessen hyperinflation, improve respiratory muscle performance, optimize thoracoabdominal mobility, and reduce dyspnea [12]. Breathing exercises performed during exercise may have various clinical benefits compared to breathing exercises performed only at rest [12]. They can promote lung function and trunk stability through diaphragmatic respiration [13].
The electrical activity of active nerve cells in the brain produces currents spreading through the head. These currents also reach the scalp surface, and resulting voltage differences on the scalp can be recorded as the electroencephalogram (EEG) [14], so we can also explore the relationship between different head postures and electroencephalogram. Many scientific studies have shown that a correct body posture is important for the best functioning of the respiratory system [15]. It can incorporate cervical stabilization exercises in the management of subjects suffering from FHP to redress faulty mechanics, which otherwise lead to impaired respiration [16].

Although there have been many previous studies on FHP, there has never been a direct comparison between cervical stabilization exercises with breathing exercises and cervical stabilization exercises with thoracic spine extension exercises in individuals with FHP.

\section{Methods}

\subsection{Participants}

The number of subjects was calculated by $\mathrm{G}^{*}$ power analysis software (ver. 3.1.9.7, Franz Faul, Kiel, GER). The sample size was calculated with 0.05 alpha level, 0.5 effect size and power of $85 \%$. This result indicated that a necessary sample size was at least 29 subjects for my study. This study included 30 subjects with FHP attending D University in Gyeongsan, South Korea. Before participating in the study, all the subjects read and signed the universityapproved human subjects consent form. Table 1 summarizes the general characteristics of the participants. The investigation was approved by the Institutional Review Board of Daegu University (IRB: \#1040621-202007-HR-002). 
The inclusion criteria employed were (1) CVA 53

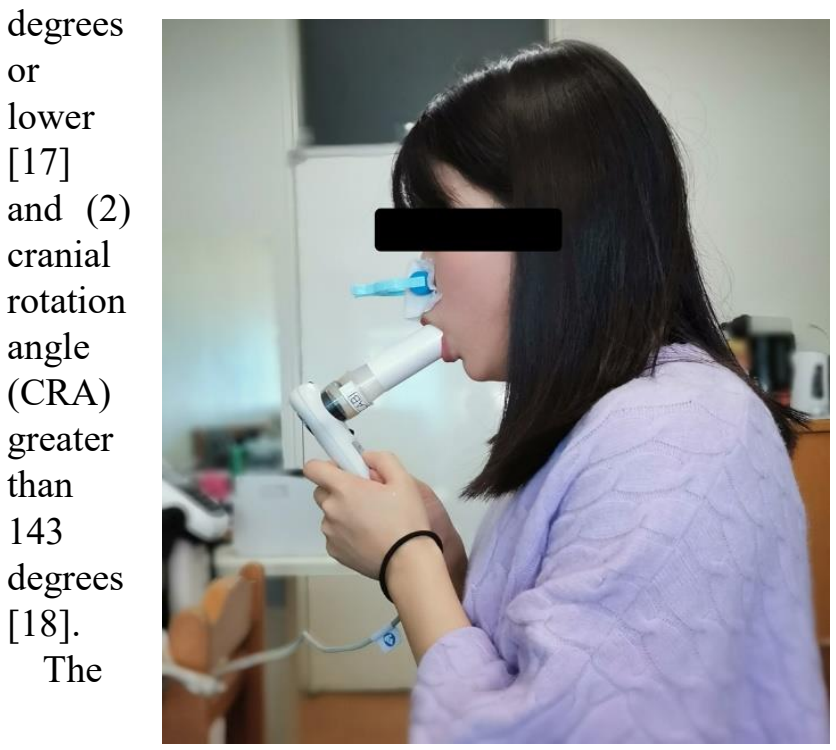

exclusion criteria were (1) mental or cognitive problems that might affect the experiment, (2) subjects with a history of cervical surgery, injury limiting activities, and cervical or thoracic fractures and (3) patients with a history of respiratory disease, such as repeated bronchitis, pneumonia, asthma, pulmonary lymph nodes, pleurisy, cured tuberculosis and others.

\subsection{Procedures}

The preparatory experiment began before the formal experiment. The study lasted from September to November 2020.

A total of 30 subjects with FHP were selected for the study. They were randomly divided into two groups, experimental (EG) and control (CG), with 15 participants in each group. The EG performed cervical stabilization exercises with breathing exercises, and the CG performed cervical stabilization exercises with thoracic spine extension exercises. Their respiratory function was measured. After 4 weeks of exercise, the respiratory function was measured again.

\subsection{Assessment}

A computer-based spirometer OMNIA software (ver. 1.6.2 COSMED, Rome, Italy) was used to measure respiratory function. Microquark USB standard package includes microQuark USB unit, turbine, nose clip, PC software, adult paper mouthpiece, pediatric mouthpiece adapter, and user manual. The function of this equipment is to formulate a diagnosis of pulmonary pathology, carry out research on human
Table 1. General characteristics of subjects

\begin{tabular}{llll} 
& & & $(\mathrm{N}=30)$ \\
\hline & $\mathbf{E G}(\mathbf{n}=15)$ & $\mathbf{C G}(\mathbf{n}=15)$ & $\boldsymbol{P}$ \\
\hline Age(year) & $22.20 \pm 2.81^{\alpha}$ & $20.53 \pm 1.92$ & .07 \\
Sex(male/female) & $7 / 8$ & $8 / 7$ & .726 \\
Height $(\mathrm{cm})$ & $170.60 \pm 9.62$ & $173.23 \pm 9.79$ & .464 \\
Weight $(\mathrm{kg})$ & $64.53 \pm 16.58$ & $68.00 \pm 15.55$ & .56 \\
BMI $\left(\mathrm{kg} / \mathrm{m}^{2}\right)$ & $21.90 \pm 3.78$ & $22.46 \pm 3.43$ & .674
\end{tabular}

EG: cervical stabilization exercises with breathing exercises group CG: cervical stabilization exercises with thoracic spine extension exercises group

${ }^{\mathrm{a}}$ mean \pm standard deviation

${ }^{*} p<0.05$

physiology, carry out research on human physiology, and get information from sports medicine. Before the test, the researcher showed the subjects how to accurately do the test two or three times in order for them to adjust to the method. To test pulmonary function, forced expiratory volume in 1 second (FEV1) and forced vital capacity (FVC) were measured. The subjects performed three familiarization trials before measurements. This was done according to Obayashi et al [19]. The measurement of respiratory function was adopted from previous studies [6]. The subjects are asked to perform a rapid full inspiration through the mouthpiece and then, without hesitation, perform a

Fig. 1 Respiratory function measurement posture

full expiration with maximum force, followed by another rapid maximum inspiration (Fig 1).

\subsection{Intervention}

\subsubsection{Cervical stabilization exercises}

With the change of time, gradually increase the difficulty of movement, is more conducive to achieve the effect of movement. Cervical stabilization exercises comprised two stages: (i) 1-2 weeks in the first stage and (ii) 3-4 weeks in the second stage.

The first stage included ligament nuchae stretching, upper trapezius stretching, levator scapular stretching, scalene stretching, and deep neck flexor strengthening (in supine and sitting positions). The second stage included deep neck flexor strengthening (in supine and sitting positions), nodding exercise, pectoralis major stretching, and 
scapular upward rotation [20]. After each exercise for 30 seconds, to prevent exercise fatigue, rest for 10 seconds, seven kinds of movements were performed once as a group, two groups at a time, three times a the foam roller on the limited spine segment, bend the knee to lie down, put both hands on the chest, gently lift a little from the floor, and slowly roll the foam roller up and down in segmentation.

Table 2. Comparison for respiratory function between two groups.

\begin{tabular}{lllllll}
\hline & & Pre & post & t & p & DV \\
\hline FVC(L) & EG & $4.07 \pm 1.13^{\alpha}$ & $4.34 \pm 1.16$ & -4.608 & $.001^{*}$ & $0.35 \pm 0.23$ \\
& CG & $4.51 \pm 1.20$ & $4.65 \pm 1.20$ & -3.192 & $.008^{*}$ & $0.09 \pm 0.13$ \\
& $\mathrm{t}$ & & & & & 3.538 \\
FEV1(L) & $\mathrm{p}$ & & & & & $.002^{*}$ \\
& EG & $3.06 \pm 0.76$ & $3.53 \pm 0.75$ & -5.409 & $.000^{*}$ & $0.54 \pm 0.31$ \\
& CG & $3.65 \pm 0.83$ & $3.88 \pm 0.90$ & -3.365 & $.006^{*}$ & $0.15 \pm 0.18$ \\
& $\mathrm{t}$ & & & & & 3.948 \\
PEF(L/s) & $\mathrm{p}$ & & & & & $.001^{*}$ \\
& EG & $5.33 \pm 1.75$ & $6.73 \pm 1.75$ & -6.727 & $.000^{*}$ & $1.45 \pm 0.68$ \\
& $\mathrm{CG}$ & $6.45 \pm 2.17$ & $7.06 \pm 2.16$ & -1.957 & .074 & $0.13 \pm 1.11$ \\
& $\mathrm{t}$ & & & & & 3.67 \\
FVC/Vcmax(\%) & $\mathrm{p}$ & & & & & $.001^{*}$ \\
& $\mathrm{EG}$ & $77.88 \pm 7.63$ & $84.47 \pm 8.04$ & -5.715 & $.000^{*}$ & $6.58 \pm 4.15$ \\
& $\mathrm{CG}$ & $80.75 \pm 4.22$ & $83.91 \pm 5.25$ & -2.838 & $.015^{*}$ & $2.19 \pm 2.63$ \\
& $\mathrm{t}$ & & & & & 3.219 \\
& $\mathrm{p}$ & & & & & $.004^{*}$ \\
\hline
\end{tabular}

FVC: Forced Vital Capacity

FEV1: Forced expiratory volume in 1 second

PEF: Peak Expiratory Flow

FVC/Vcmax\%: Forced Vital Capacity/Vital Capacity max

EG: cervical stabilization exercises with breathing exercises group

CG: cervical stabilization exercises with thoracic spine extension exercises group

DV: different value

${ }^{\mathrm{a}}$ mean \pm standard deviation

$* p<0.05$

week for 4 weeks.

\subsubsection{Breathing exercises}

Breathing exercises were adopted from previous studies [21]. They included breathing awareness, costal expansion, diaphragmatic breathing exercises (in supine and prone positions), and diaphragmatic breathing exercises in supine position with a $1 \mathrm{~kg}$ weight on the diaphragm in the abdominal region. Each exercise lasted for 3 minutes and was repeated twice, three times a week for 4 weeks.

\subsubsection{Thoracic spine extension exercises}

Thoracic spine extension exercises were taken from previous studies [10]. Each exercise was performed 15 times as a group and repeated for the two groups three times a week for 4 weeks:

-Thoracic spine extension exercise 1 program: Put
- Thoracic spine extension exercise 2 program: In order to protect the knee, lay a mat on the ground, bend the knee, put the Swiss ball, hands on the ball, and walk forward. The upper body is tilted forward to debilitate the upper spine, which is maintained at the end point for 10 seconds.

-Thoracic spine extension exercise 3 program: With both arms supported, lift the upper body up repeatedly as in the existing demotion position. The patients were maintained in the demeaning position for 7 seconds.

\section{Statistical analysis}

The Kolmogorov-Smirnov test was used for a normal distribution. The paired t-test was used to compare the difference within the group before and after the intervention of the two groups, and the 
independent $\mathrm{t}$-test was used to determine the difference between two groups. Statistical analyses were performed using SPSS (Statistical package for the social sciences) version 20.0 for Window software (SPSS, IBM, USA), and the statistical significance level was set at $\alpha=0.05$.

\section{Results}

According to statistics from the paired t-test, after the exercises, FVC, FEV1, peak expiratory flow (PEF), and $\mathrm{FVC} / \mathrm{VCmax} \%$ were found to be significantly different in the EG $(p<0.05)$, and FVC, FEV1, and $\mathrm{FVC} / \mathrm{VCmax} \%$ were significantly different in the CG $(\mathrm{p}<0.05)$ (Table 2).

On the basis of the statistics from the independent $\mathrm{t}$-test, after the exercises, significant differences were noted in the values of FVC, FEV1, PEF, and $\mathrm{FVC} / \mathrm{VCmax} \%$ between the two groups $(\mathrm{p}<0.05)$ (Table 2).

\section{Discussion}

FHP is becoming increasingly common in day-to-day life, affecting respiratory function. This study aimed to explore the effects of exercises on respiratory function.

The results showed statistically significant differences in the EG and CG before and after the intervention. In this study, both groups performed cervical stabilization exercises. Sonia et al. [16] reported that a reduction in FHP by performing cervical stabilization exercises improves respiratory function by correcting the altered biomechanics of the cervical and thoracic spine, which improves thoracoabdominal mobility, and the efficacy of the diaphragm can be improved.

The exercises of the EG involved a lot of neck and shoulder muscles. Kang et al. [10] found that since the neck and shoulder muscles directly take part in breathing, their alignment enables optimum respiratory function. However, in the between-group comparison, different values of respiratory function had significant differences. These differences were greater in the EG than in the CG. In the EG, diaphragmatic breathing exercises and inspiratory muscle training were included in the breathing exercises program followed in this study. Borge et al. [22] reported that diaphragmatic breathing exercises focus on minimizing the actions of accessory muscles, activating the diaphragm during inspiration. Respiratory muscle training improved the strength and endurance of the respiratory muscles [23-25].
Kong et al. [26] demonstrated that cervical exercises were performed for only relatively for 4 weeks, the exercises caused an improvement in the forward head posture. Yong et al. [13] reported that diaphragmatic breathing exercises promote lung function and trunk stability. After 4 weeks of exercise, respiratory function showed a significant improvement.

However, the limitations of this study should also be noted. First, the experimental period was short. Second, except for the exercise time in the laboratory, the other exercise times could not be accurately controlled. Third, the leisure sports activities of the subjects might have affected the experimental results. Fourth, the study population included only students, not other professional groups. Future studies may wish to consider a long experimental period, increase the number of people with different occupations, and accurately control other types of sports.

\section{Conclusion}

This study aimed to compare the effects of cervical stabilization exercises with breathing exercises and cervical stabilization exercises with thoracic spine extension exercises on respiratory function in subjects with FHP. Respiratory function was found to be significantly improved in the EG. Hence, cervical stabilization exercises with breathing exercises are recommended to promote postural alignment and respiratory function.

\section{ACKNOWLEDGEMENT}

This research was supported by the Daegu University Research Grant, 2020.

\section{Reference}

[1] Zafar, H., Albarrati, A., Alghadir, A. H., Iqbal, Z. A, Effect of Different Head-Neck Postures on the Respiratory Function in Healthy Males, BioMed research international, 2018, pp. 1-4.

[2] Cho, J., Lee, E., Lee, S, Upper cervical and upper thoracic spine mobilization versus deep cervical flexors exercise in individuals with forward head posture: A randomized clinical trial investigating their effectiveness, Journal of back and musculoskeletal rehabilitation, Vol. 32, No. 4, 2019 , pp. 595-602.

[3] Hajar Razaghi., Reza Saatchi., Nick. J. Bishop., Derek Burke., Amaka C. Offiah, Evaluation of Vibration Analysis to Assess Bone Mineral 
Density in Children, WSEAS Transactions on Biology and Biomedicine, x Volume 17, 2020, pp. 48-57.

[4] Kim, S. Y., Kim, N. S., Kim, L. J, Effects of cervical sustained natural apophyseal glide on forward head posture and respiratory function, Journal of physical therapy science, Vol. 27, No. 6, 2015, pp. 1851-1854.

[5] Kong, Y. S., Kim, Y. M., Shim, J. M, The effect of modified cervical exercise on smartphone users with forward head posture, Journal of physical therapy science, Vol. 29, No. 2, 2017, pp. 328-331.

[6] Han, J., Park, S., Kim, Y., Choi, Y., Lyu, H, Effects of forward head posture on forced vital capacity and respiratory muscles activity, Journal of physical therapy science, Vol. 28, No. 1, 2016, pp. 128-131.

[7] Kim, M. S., Cha, Y. J., Choi, J. D, Correlation between forward head posture, respiratory functions, and respiratory accessory muscles in young adults, Journal of back and musculoskeletal rehabilitation, Vol. 30, No. 4, 2017, pp. 711-715.

[8] Okuro, R. T., Morcillo, A. M., Ribeiro, M. Â., Sakano, E., Conti, P. B., Ribeiro, J. D, Mouth breathing and forward head posture: effects on respiratory biomechanics and exercise capacity in children, Jornal brasileiro de pneumologia: publicacao oficial da Sociedade Brasileira de Pneumologia e Tisilogia, Vol. 37, No. 4, 2011, pp. 471-479.

[9] Wirth, B., Amstalden, M., Perk, M., Boutellier, U., Humphreys, B. K, Respiratory dysfunction in patients with chronic neck pain - influence of thoracic spine and chest mobility, Manual therapy, Vol. 19, No. 5, 2014, pp. 440-444.

[10] Kang, J. I., Jeong, D. K., Choi, H, Correlation between pulmonary functions and respiratory muscle activity in patients with forward head posture, Journal of physical therapy science, Vol. 30, No. 1, 2018, pp. 132-135.

[11] Charalampos Platis., Adamantia Spanou., Pantelis Messaropoulos., Catherine Kastanioti., Emmanouil A. Zoulias, Diabetic Patients' Quality of Life and their Relationship in Compliance with Antidiabetic Treatment. Case Study of Patients in a Public Hospital in Greece, WSEAS Transactions on Biology and Biomedicine, Volume 17, 2020, pp. 32-38.
[12] Holland, A. E., Hill, C. J., Jones, A. Y., McDonald, C. F, Breathing exercises for chronic obstructive pulmonary disease, The Cochrane database of systematic reviews, 2012.

[13] Yong, M. S., Lee, H. Y., Lee, Y. S, Effects of diaphragm breathing exercise and feedback breathing exercise on pulmonary function in healthy adults, Journal of physical therapy science, Vol. 29, No. 1, 2017, pp. 85-87.

[14] Ayman, M., Mansour., Mohammad A. Obeidat., Murad Al-Aqtash, Data Mining based Approach for Evaluation of EEG Signals for Epilepsy Detection, WSEAS Transactions on Biology and Biomedicine, Volume 17, 2020, pp. 48-57.

[15] Szczygieł, E., Węglarz, K., Piotrowski, K., Mazur, T., Mętel, S., \& Golec, J, Biomechanical influences on head posture and the respiratory movements of the chest, Acta of bioengineering and biomechanics, Vol. 17, No. 2, 2015, pp. 143-148.

[16] Pawaria, S., SuDhan, D. S., Kalra, S, Effectiveness of cervical stabilization exercises with feedback on respiratory status in chronic neck pain patients with forward head posture, International Journal of Physiotherapy, Vol. 6, No. 3, 2019, pp. 70-75.

[17] Lee, D. Y., Nam, C. W., Sung, Y. B., Kim, K., Lee, H. Y, Changes in rounded shoulder posture and forward head posture according to exercise methods, Journal of physical therapy science, Vol. 29, No. 10, 2017, pp. 1824-1827.

[18] Kim, E. K., Kang, J. H., Lee, H. T, The effect of the shoulder stability exercise using resistant vibration stimulus on forward head posture and muscle activity, Journal of physical therapy science, Vol. 28, No. 11, 2016, pp. 3070-3073.

[19] Obayashi, H., Urabe, Y., Yamanaka, Y., Okuma, R, Effects of respiratory-muscle exercise on spinal curvature, Journal of sport rehabilitation, Vol. 21, No. 1, 2012, pp. 63-68.

[20] Sahrmann, S, Movement system impairment syndromes of the extremities, cervical, and thoracic spines, Elsevier/Mosby, 2011.

[21] Tomas-Carus, P., Branco, J. C., Raimundo, A., Parraca, J. A., Batalha, N., Biehl-Printes, C, Breathing Exercises Must Be a Real and Effective Intervention to Consider in Women with Fibromyalgia: A Pilot Randomized Controlled Trial, Journal of alternative and complementary medicine (New York, N.Y.), Vol. 24, Vo. 8, 2018, pp. 825-832. 
[22] Borge, C. R., Hagen, K. B., Mengshoel, A. M., Omenaas, E., Moum, T., Wahl, A. K, Effects of controlled breathing exercises and respiratory muscle training in people with chronic obstructive pulmonary disease: results from evaluating the quality of evidence in systematic reviews, BMC pulmonary medicine, Vol. 14, No. 1, 2014, pp. 1-15.

[23] Lnger, D., Ciavaglia, C., Faisal, A., Webb, K. A., Neder, J. A., Gosselink, R., Dacha, S., Topalovic, M., Ivanova, A., O'Donnell, D. E, Inspiratory muscle training reduces diaphragm activation and dyspnea during exercise in COPD, Journal of applied physiology (Bethesda, Md. : 1985), Vol. 125, No.2, 2018, pp. 381-392.
[24] Liaw, M. Y., Hsu, C. H., Leong, C. P., Liao, C. Y., Wang, L. Y., Lu, C. H., Lin, M. C, Respiratory muscle training in stroke patients with respiratory muscle weakness, dysphagia, and dysarthria - a prospective randomized trial, Medicine, Vol. 99, No. 10, 2020, pp. 1-10.

[25] Menezes, K. K., Nascimento, L. R., Avelino, P. R., Alvarenga, M., Teixeira-Salmela, L. F, Efficacy of Interventions to Improve Respiratory Function After Stroke, Respiratory care, Vol. 63, No. 7, 2018, pp. 920-933.

[26] Kong, Y. S, Kim, Y. M, Shim, J. M, The effect of modified cervical exercise on smartphone users with forward head posture, J Phys Ther Sci, Vol. 29, No. 2, 2017, pp. 328-3

\section{Creative Commons Attribution License 4.0 (Attribution 4.0 International, CC BY 4.0)}

This article is published under the terms of the Creative Commons Attribution License 4.0

https://creativecommons.org/licenses/by/4.0/deed.en_US 